chemist. Theoretical contributions to this branch of science are comprised in a series of papers on "The Possible Significance of Changing Atomic Volume," in which he suggests a relation between the energy of the atoms and their compressibilities. In order to test his hypothesis, he has made a long series of investigations on the compressibility of elements and compounds. He has determined this constant for nearly all the solid and liquid elements, and he has shown that the compressibility is a periodic function of the atomic weights. In electrochemistry Prof. Richards has made important determinations of the electro-chemical equivalent of silver, and he has supplied some of the most rigorous proofs of the universality of Faraday's law.

\section{Darwin Medal.}

To Mr. Roland Trimen, who was for many years curator of the South African Museum in Cape Town, the Darwin medal has been awarded. His official position, and the duties it involved, enabled him to do admirable work in African zoology. His name will always stand with those of Bates and Wallace in the establishment and illustration of the theory of mimicry. In addition to his researches on that subject, he has done admirable systematic work, his descriptions of insects, especially the Lepidoptera rhopalocera, being models of accuracy and literary style. He, furthermore, rendered the greatest assistance to Charles Darwin, especially in his work on orchids-assistance the high value of which is acknowledged in a long series of that great naturalist's published letters.

\section{Sylvester Medal.}

The medal which perpetuates the name and mathematical prowess of James Joseph Sylvester has this year been assigned to Dr. Henry Frederick Baker, in recognition of his work in the theory of functions, wherein he has shown himself to be a profound analyst. His book on the Abelian functions, published in 1897 , is a classic, and probably no better guide to the analytical development of pure mathematics has appeared during the last three-quarters of a century. While basing the argument of the work on the methods of Riemann, he never loses sight of the arithmetical ideas which we owe to Kronecker, Dedekind, and Weber, or of the geometrical notions brought to light by the labours of Clebsch, Gordan, Noether, and Klein. The critical insight which was thus in evidence marked him out a few years ago as the editor of "Sylvester's Collected Papers." "This work, which, with the approaching issue of the fourth and last volume, may be said to be complete, has been necessarily a difficult task, which, besides making demands upon the resources of an accomplished mathematician, has entailed no little editorial labour. Dr. Baker, by explanatory and critical observations, and by frequent ameliorations of the text, has done much to assist mathematical students. His scholarly work has resulted in a faithful record of the course of Sylvester's thought. It seems eminently fitting that the Sylvester medal should be given to one who has erected so lasting a memorial to the great mathematician.

\section{Hughes MEdal.}

To Prof. John Ambrose Fleming the Hughes medal has been awarded. For thirty years he has been actively engaged in researches in experimental physics, chiefly in the technical applications of electricity. He was an early investigator of the properties of the glow lamp, and elucidated the unilateral conductivity presented in its partial vacuum between glowing carbon and adjacent metal, a phenomenon which has been linked up recently with the important subject of the specific discharges of electrons by different materials. He has published in the scientific and technical Press, and in technical text-books, many admirable experimental investigations and valuable expositions in the applications of electricity, as, for example, to electric transformers and wireless telegraphy. Of special interest and value for theory were the important results concerning the alterations in the physical properties of matter, such as the remarkable increase in the electric conductivity of metals when subjected to very low temperatures, which flowed from his early collaboration with Sir James Dewar in investigating this domain. In recent years he has taken a prominent part in the scientific development of telegraphy by free electric waves.

In the evening the fellows and their guests dined together at the Whitehall Rooms, Hotel Metropole.

\section{ENTERIC FEVER CARRIERS.}

$T \mathrm{HE}$ frequent difficulty in accounting for the source of inrection of enteric fever once led to the theory that this disease could arise de novo, that is to say, that certain organisms in human dejecta were capable of developing, in favourable circumstances, into enteric fever organisms. It has also been maintained more recently that the specific organism of this disease was capable of living and multıplying in water and soil, for considerable periods. But the bacteriological work of the past few years has discredited both these hypotheses; and the "carrier" case of enteric fever or the mild, unrecognised case of infection generally, explain the transmission of the disease in those cases in which the disease crops up in the absence of any recognised sufferer from the disease.

A "carrier" of enteric fever is a person who, although he may be in good health, carries the infectious material in his body, from which it may pass out. He is not merely a passive transmitter of infection; he is also a breeding-ground and storehouse of these specific organisms; and it appears that not only those sick with the fever, but also healthy persons who happen to be "carriers" of the infection, offer the best explanation for the maintenance of the infection in communities.

The subject has naturally attracted much attention and led to many investigations, the results of which are to be found in numerous recent publications, and Dr. Ledingham has done a great service in preparing for publication a summary of the more important investigations that have hitherto been made of this subject. He gives the history of a large number of occurrences of enteric fever in domestic life, in institutions, and in military populations, in which the source of infection has been traced more or less convincingly to a "carrier." In many of these cases the evidence is conclusive that the infection was conveyed by food or milk. The recorded instances go to prove that the female sex is more liable to carry the infection than the male, and that of both sexes some 2 to 4 per cent. of previous sufferers may continue to harbour the germ, and become "carriers," who intermittently discharge the germ, for periods extending maybe for many years.

As Dr. Theodore Thomson, who writes an introduction to this report, states, the difficulty of dealing with "carriers" is very great indeed, having regard more particularly to the long periods during which people may harbour the infection and to the fact that it has hitherto proved very difficult to free them from the infection. The chief available measures include : all possible efforts to detect "carriers" in the community, and to endeavour to secure on the part of a "carrier" those precautions of strict personal cleanliness and of disposal of dejecta that will minimise the risk of infecting others; an endeavour must also be made to prevent such "carriers" from taking anv part in the milk trade or in the preparation or handling of food.

In this interesting report, Dr. Ledingham also discusses the diagnostic methods employed in the search for "carriers" and the immunity question in "carriers." A useful bibliography is appended.

1 Dr. J. C. G. Ledingham's Report to the Local Government Board on subject. Pp. $x_{3} 8$. (London: Wyman and Sons, 1910.$)$ Price $1 s$. 